\title{
Pro-Tempore Disjunctive Intentions
}

\author{
Luca Ferrero \\ Philosophy \\ UW-Milwaukee \\ PO Box 413 \\ Milwaukee, WI 53201-6413 \\ luca.ferrero@gmail.com \\ http://people.uwm.edu/ferrero
}

26 Apr 2016

Version 1.62 forthcoming in R. Altshuler and M.J. Sigrist (eds.) Time and The

Philosophy of Action, Routledge 


\title{
Pro-Tempore Disjunctive Intentions
}

\author{
Luca Ferrero
}

\section{Introduction}

1.1 In planning for the future, we face two basic challenges. First, we must organize our steps to deal with the uneven temporal distribution of the opportunities and resources to make progress in ourpursuits. Second, we need to deal with our limited knowledge of the future and with the changes that this knowledge undergoes over time. We hardly ever plan while being certain of all the relevant future circumstances.

Our intentions have a built-in protection against the unexpected: it is in principle always possible to give up an intention in the face of unanticipated future circumstances. Yet this shouldbe a strategy of last resort, to be used sparingly and judiciously. Over-reliance on changes of mind, even when justified, would undermine the stability of intentions, which is a distinctive feature of the standard operation of intentions and a major source of the appeal of planning abilities.

1.2 Our plans need to balance stability and flexibility. We must avoid two opposite responses to uncertainty and risk, just giving up the intention altogether as we come to learn more, or a reluctance to undertake any plan at all when we are not certain about the future.

There are two obvious strategies to make intentions more flexible, while preserving their stability. First, as Bratman (1987) shows, our plans are partial: they need not specify all the details of implementation. We make appropriate adjustments as we progress and acquire relevant knowledge.

Second, most intentions are conditional. Even when expressed categorically, as intentions to $\varphi$, we are not usually committed to $\varphi$ ing no matter what. Most ordinary intentions are implicitly conditional upon circumstances we are uncertain about - they are of the form "I intend to $\varphi$ if $C$," where $C$ has not yet been 
ascertained by us. ${ }^{1}$

1.3 Here I present an additional strategy: the contemporaneous pursuit for the time being of two or more eventually incompatible projects. Consider a not yet fully resolved traveling plan: I am settled on either going to a conference in Italy or to a festival in Austria. The two projects are incompatible because the events are contemporaneous. Although I have not yet made up my mind between the two, I have already determined that I am going to one of them. Hence I can legitimately say: "I intend to go either to Italy or to Austria."

Let's call these intentions pro-tempore disjunctive (PTD, for short). They are usually undertaken when we don't yet know which alternative is preferable while expecting that we will gain relevant information later, at which point we will continue to pursue only one of the alternatives.

1.4 PTD intentions are a familiar strategy to trade off stability and flexibility. But one that has been neglected by philosophers, with the exception of Holton (2009). They are worth of a closer look. There is much that we can learn from them about the nature and dynamics of planning agency.

I first discuss simple disjunctive intentions, where one is indifferent between the alternatives. I then show that PTD intentions are different from simple disjunctive intentions. I discuss how PTD intentions meet the rational pressures of intentions and how they settle practical matters. PTD intentions are a pervasive feature of diachronic agency: they are the simplest tools to agglomerate potentially conflicting pursuits without rejecting them outright. They are the basic strategy for balancing both rigidity and flexibility, and stability and responsiveness to changing circumstances. I close by rejecting Holton's partial intentions. We should not introduce a novel kind of attitude: all-out intentions with complex contents, such as PTD, can do all the necessary work.

2 Disjunction of Indifference 
2.1 When one is indifferent between two incompatible courses of action, one can acquire a disjunctive intention of indifference. Someone approaches me with a tray with two cookies. I only want one and I am indifferent between them. I just need to form the intention to either get cookie $A$ or get cookie $B$, that is, to make true the disjunction (get $A \vee$ get $B$ ). Because of my indifference, I need not be more specific. No need to settle for 'getting the closest cookie,' say. After all, by the time I reach the tray, the cookies might be equidistant from me. It only matters that I get one.

2.2 Disjunctive plans of indifference are common. But their incompleteness might be troubling. An intention is supposed to settle what to do, but a disjunctive plan leaves open what the agent is to do once she reaches the "conflict point" (c-point, hereafter) where the two alternatives become incompatible. Even so, a disjunctive intention has practical import. Prior to the cpoint, it guides one's conduct to reach the c-point (i.e., to get within the reach of the cookies). And it continues to guide even at the c-point by telling what not to do then: not to refrain from reaching for one of the cookies. It directs the agent away from the complement of the disjunction, which is the practical import that Buridan's ass missed.

The disjunctive intention does not tell one which cookie to pick. But this is unproblematic. The intention fulfills its guiding role as long as it secure the presence, at c-point, of some mechanism that moves one to pick one cookie. The resolution of this practical underdetermination does not need to come from an intention with a non-disjunctive content (i.e., directed at a specific cookie). The intention is only for making a disjunction true, which is something that at c-point can be accomplished by whatever psychological or physiological mechanism might make one grab a cookie.

2.3 To sum up, given one's indifference, the job of an intention as a planning attitude, is fully discharged by a purely disjunctive intention to (A $\vee$ B). This intention is fully adequate to discharge its characteristic job: settling on and stably guiding future conduct, framing further deliberation, and coordinating conduct transtemporally and interpersonally. 
This intention needs to be paired with the power to pick one disjunct. But this is just one of the fundamental executive capacities that all agents need in order to carry out any of their intentions.

2.4 Purely disjunctive intentions are familiar but they apply to a limited range of circumstances. An intention to $(A \vee B)$ is adequate when one is indifferent between $A$ and $B$ but the two options do not call for distinctive preparations and the choice between them does not have distinct long-term effects. In the cookie example, no specific preparation is required. Upstream of the c-point, one is only required to keep the possibility of future choice open. And once the choice is made, one does not expect it to have divergent downstream effects.

2.5 This analysis applies also to cases of normative underdetermination (including those due to incommensurability and parity). When facing the prospect of two incompatible options that one cannot rank, as far as planning is concerned, one might simply settle for making their disjunction true. This is not to deny that, given the specific source of the underdetermination, the agent might have other resources that could help choose or pick between the options when she reaches the c-point. But the resources that come from intentions as planning attitudes run out in the face of this underdetermination, if one anticipates that the underdetermination is going to persist up to the c-point. This is so even if one takes into account the needs for advanced coordination with one's future choice between the two options.

Obviously, the choice at the c-point is much more momentous in cases of underdetermination than in those of mere indifference, including the potential for massively divergent downstream effects. But in a genuinely persistent underdetermination, even the anticipation of divergent downstream effects cannot make a difference to what the planning capacities might contribute. The best we can do is to settle for making a disjunction true. ${ }^{2}$

2.6 A disjunctive intention puts no pressure on keeping the options open. If the opportunity to carry it out by going for one of the disjuncts arises earlier than the anticipated c-point, one 
might be strongly advised to take it (but not required to do so, if the opportunity for future success remains available). Likewise, if one expects future success in executing one of the disjuncts, it is permissible to let go of taking means necessary for the pursuit of the other. Nonetheless, if the disjuncts do not make distinct and costly preparatory demands, one might keep both open until the c-point. Incommensurability over momentous choices might favor delaying the choice but this delay is rationally demanded only if one suspects that additional information might become available and remove the incommensurability.

\section{Pro-Tempore Disjunctive Intentions}

3.1 Let's return to the original scenario about my summer vacation and consider how it differs from a plan to make a disjunction true. First, I am not indifferent between Italy and Austria. It is rather that I am presently unable to tell which among these two incompatible options is better. I expect to be in a position to rank them later on account of either new information or the additional time I can devote to deliberation. I might even expect one of the two options to turn especially bad, possibly one to be avoided at all costs (if, for instance, I were to learn that my archenemy is expected to be at one of those locations). Nonetheless, I have already determined that I should restrict my travel options to these two locations and I am already under a pressure to start making the appropriate arrangements.

In this scenario, I am not intending to make the disjunction (going to Italy $\vee$ going to Austria) true, as if I were indifferent between the options. In particular, I am committed to keeping them open for the time being, since I do not yet know which one will turn out to be preferable at a later time. This makes a real difference if, as it is often the case, the preparatory arrangements for the two options are substantially different. When so, to keep both of them open carries a price. In addition to the distinct preparatory steps, I have to pay various costs, including those associated with the delaying of the final determination and with the forgoing of opportunities for 
alternative investments. But the circumstances might be such that it would still be reasonable for me to incur these costs, rather than risking to make either option impossible or being forced to make an early choice under more limited information.

The plan is not to leave the matter unresolved, in an unattended manner. This strategy might be best in those cases where delaying the preparation carries limited costs, if any. But this is not so in the present scenario. Hence, the plan is not to let the events run their course, make no commitment, and revisit the question later at a hopefully more favorable time. Here I am rather undertaking the intention to sustain the viability of the options until I am in a better position to make a choice, either because I will have gained more relevant information or I will face an unavoidable c-point.

3.2 What I have is an intention. It is the intention to sustain the viability of two options (Italy and Austria) for the time being in spite of their eventual incompatibility. This is not a case of simply intending to make a disjunction true. It is rather a plan that comes in two stages: at the earlier stage, while the implementation of both options is still possible and I am still waiting to determine which is best, I am pursuing both. At the later stage, once I have determined which is best or I am faced with an inevitable cpoint, I only pursue one (although, prior to that moment, I do not yetknow which one). This is a familiar kind of plan, whose form underlies many of our disjunctive expressions of intention. Oftentimes, when we say that we intend to do either Aor B, we are not expressing indifference but rather a pro tempore disjunctive intention.

Here is a more formal characterization of the content of a protempore disjunctive intention (PTD intention). Given two eventually co-impossible goals $A$ and $B$, let's call c-point the time when the two goals inevitably conflict and it is no longer possible to continue to pursue both; let's call $d$-point the moment prior to (and inclusive of) the c-point when the subject determines, if ever, which option is better than the other.

A pro-tempore disjunctive intention to pursue either $A$ or $B$ is the intention: 
1. to pursue $A$ at least until d-point (or until c-point if there is no d-point), and

2. to purse $B$ at least until d-point (or until c-point if there is no d-point), and

3. to continue pursing $A$ past d-point only if $A$ is deemed better than $B$ at that time, and

4. to continue pursuing $B$ past d-point only if $B$ is deemed better than $A$ at that time, and

5. to purse $(\mathrm{A} \vee \mathrm{B})$ as a matter of indifference at c-point if there has been no prior or contemporaneous d-point, ${ }^{3}$

6. while believing throughout that $A$ and $B$ are coimpossible past the c-point.

3.3 Because of the two stages, a PTD intention does not run afoul of the demand for agglomerativity. As Bratman $(1987,134)$ has compellingly argued, "given the role of intentions in coordination, there is a rational pressure for an agent to put his various intentions together into a larger intention." The "larger intention" has usually been interpreted as the conjunction of the objects of the agent's intentions. For instance, if I intend to go to Italy and I intend to go to Austria, I am under a rational pressure to intend to go both to Italy and to Austria. ${ }^{4}$

The principle of agglomerativity formalizes the intuitive idea that a rational agent is not to embark on a self-defeating course of action. The rational agent must avoid the contemporaneous pursuit of co-impossible goals. But this does not entail that the agent is to give up one of the goals altogether, at least not from the very beginning. Given that the pursuits are co-impossible only past the c-point, it is not irrational to continue pursuing both, up until that point, in the form of a PTD plan. That is, with the knowledge that one is going to face a choice between them no later than at c-point.

3.4 The mutual consistency among one's plans is something to be achieved dynamically, as one progresses in their pursuit and one acquires (and sometimes loses) additional relevant information.

The simplest method to secure consistency is to give up one 
of the co-impossible goals. But this might be too radical and premature a response, especially if one expects to gain relevant information later.

Another useful strategy is to subordinate one pursuit to the other. I might settle on Italy and intend to go to Austria only if going to Italy becomes impossible or unadvisable. When so, I am no longer under a demand to take steps to go to Austria, as long as the Italy option is still feasible. Hence, I take on the risk of incurring additional costs if going to Italy turns out later to be unfeasible or unadvisable (given that, by that time, the trip to Austria might have become much more expensive, if not impossible).

3.5 Alternatively, I could pursue both plans in a conditional form. I might list the conditions $C_{I}$ under which I intend to go to Italy, and the conditions $C_{A}$ under which I intend to go to Austria. If $C_{I}$ and $C_{A}$ are mutually exclusive, I might be able to agglomerate the two intentions without irrationality, provided that the preparatory steps do not encroach on each other (by making one pursuit possible at the expenses of the other). I still cannot expect to succeed at both, but the failure would not count as self-defeating conduct. Rather, because of the mutually exclusive conditions, at least one of the plans will turn moot once its conditions are no longer believed to be possible. ${ }^{5}$

The conditional strategy is a more sophisticated form of the dynamical consistency secured by PTD: it requires the articulation of conditions that qualify the alternative options. By comparison, a PTD intention only requires that one is committed to keeping the options open while one is trying to figure out which one is preferable. As one learns more about the alternatives, however, the PTD intention might transform into a combination of two conditional plans, with mutually exclusive antecedents. This kind of metamorphosis is part and parcel of the adjustments that a rational agent makes in securing the mutual consistency and continuous progress of her plans over time.

3.6 All these strategies for mutual consistency are in principle equally available. Which strategy is preferable depends on the specific circumstances. But the PTD strategy is always the simplest strategy to dynamically handle competing demands of 
eventually incompatible projects - short of renouncing one's goals altogether.

As the simplest strategy, it does not impose a specific structure on how the plan is to unfold. It does not articulate in advance the specific conditions under which one option rather than the other is to be pursued. Nor does it institute a structure of subordination. But it is a useful starting point, which occasionally might lead to subordination or articulation of more specific conditions. Because of its simplicity, it might be abused and become a tool for massive procrastination. An agent who is reluctant to make choices might continue to undertake more and more eventually incompatible projects in the PTD from, but later face the dreaded moments of choice at much higher costs. On the positive side, learning about PTD structure and its characteristic demands helps us become more proficient in handling the balance between fixity and stability, on the one hand, and flexibility and responsiveness to changing circumstances, on the other. It offers the basic tool to keep concurrent projects open but also to develop strategies to reduce the risks and costs associated with concurrent pursuits (for instance, by inviting us to settle on projects with a larger overlap in preparatory steps or to increase reliance on of all-purpose means).

PTD plans are the most basic tools for managing the temporal agglomeration of distinct projects and for achieving dynamic consistency by limited agents like us, who need to handle the uneven temporal distribution of resources and opportunities for action, and their limited information. It allows for the simplest, even if only temporary, integration. This integration is a better strategy than the rigidity of agents who either refuse to undertake incompatible plans altogether or undertake them while ignoring their eventual incompatibility, thereby setting themselves up for the high costs of the unanticipated later encounters with c-points.

3.7 There is still a concern with PTD plans. The characteristic benefits of planning derive from its power to settle one's conduct in advance, but a PTD intention might still leave wide open what the agent is going to do. Can one really settle a practical question in a stable manner by acquiring a PTD intention? 
Consider stability. A PTD intention is subject to the pressure for stability with the same force and character as any other intention. That a rational agent is expected to drop one of the disjuncts does not make her intention more unstable. Dropping one of the options is not the same as giving up the PTD intention altogether. Rather, it is a rational transformation that the intention is supposed to undergo as time goes by and more information is acquired. This is similar to the metamorphosis that occurs to a conditional intention, when the conditions are taken to hold and the antecedent is thereby discharged. When so, one is not giving up the original conditional intention, one rather continues to carry itout in its new categorical shape. ${ }^{6}$ Likewise, once the agent drops one disjunct, she continues to carry out the same intention in its new non-disjunctive form.

This conclusion holds for all rational pressures constitutive of intending (such as means-end coherence and agglomerativity), not just stability. They apply with the same force regardless of the content of the intention. In this sense, a conditional intention is not weaker than a categorical one. Likewise for PTD intentions, which are neither weaker nor more unstable than categorical ones. This is why an agent can be settled on a PTD intention as much as she is on a categorical one.

3.8 At least, this is so for what might be called "structural" settledness. A rational agent is structurally settled on what she is going to do in the future by way of taking a distinctive attitude - an intention - toward her future conduct. There is still a substantive question: what is she settled on? This depends on the content of the intention rather than the nature of her attitude. In the substantive sense, a PTD intention seems to determine much less about one's future conduct than a categorical intention, for much is still open about what one is going to do both before and after the c-point. This might seriously reduce the contribution of PTD intentions to transtemporal intra- and inter- personal coordination-which is a distinctive benefit of our reliance on intentions.

But PTD intentions still help with coordination. Like any intention, a PTD one restricts the range of conduct expected of a rational agent. At the very minimum, a rational agent is not to 
make impossible for her to carry out her intention. For a PTD intention, prior to c-point, the range of expected conduct might actually be more limited than for a categorical one to pursue just one disjunct. For instance, before reaching the c-point, not all the ways of my going to Italy are compatible with my going to Austria. Hence, if you need to coordinate with my position prior to the c-point, there are situations in which you might be better off if I have a PTD intention rather than an unconditional one. Prior to the c-point, there are fewer courses of action that I might rationally take in the PTD case than in the unconditional one. For prior to the c-point, I am not expected to make progress toward my going to Italy in a way incompatible with my going to Austria. When you consider coordination after the c-point, however, my PTD intention is less useful than an unconditional one, because of the divergent unfolding of the ways in which I might rationally pursue the plan that I have now chosen at the expenses of the other. In any event, even after the c-point, having a PTD intention still provides a better guide for transtemporal coordination than having no intention atall.

3.9 Coordination only requires that one be able to determine, at least roughly, the size and shape of the area where the rational agent is supposed to be on account of her intention. A rational agent is not supposed to get outside of this area, since this exit would amount to a failure of her project. This is not the same as coordinating with an agent who is settled on a very specific goal which can only be implemented in a univocal way. When so, one could anticipate each and every move of this agent and reduce the area for coordination to a single trajectory. But this is a limiting case. Much coordination, especially for the longer term, is done by securing that one stays within a certain area rather than on a specific trajectory.

The smaller the area, the easier it is to anticipate where the agent will be and to coordinate with her based on her expected future location. The extent of this area, however, is not simply a matter of the form taken by the content of the intention. A PTD intention might actually delimit a smaller area than a categorical one. After all, my PTD intention "to go either to Italy or to Austria" is more helpful, for purposes of coordination, than a non-disjunctive but more generic intention "to go to Europe." 
4 The Pervasiveness of Pro-Tempore Disjunctive Intentions

4.1 The structure of PTD intentions is not just a curiosity. It is a pervasive feature of our intentional diachronic agency. It provides the basic strategy to secure the dynamic consistency of our plans in their temporal unfolding, given that we need to respond to our previous successes and failures, and to changes in relevant information. PTD intentions are not limited to brand new plans about options we are yet unable to rank. They might also be adopted to agglomerate a new goal with some old ones. PTD intentions work as placeholders for future choices, while we continue to move on with all of our concurrent (although not all eventually co-possible) projects.

4.2 In addition, the PTD structure underlies the "partiality" of plans (see Bratman 1987). Many details about implementation are initially left unspecified and they are filled over time. As one gets closer to the need to take more specific steps, additional and more accurate information is usually more easily available to help determine the details of implementation.

Partiality is just a special instance of the PTD structure. Let's imagine that one intends to $\varphi$ at a later time $t_{3}$. Consider the step that she has to take at $t_{2}$ to progress toward her later $\varphi$-ing. This step might take very different specific shapes, $s_{1}, s_{2}, \ldots, s_{m}$. These shapes are mutually incompatible pieces of local conduct. When the agent at $t_{1}$ thinks about what she is going to do at $t_{2}$, she might be in no better position than to intend to take one of these steps, without knowing yet which one. But she is also committed to keeping them open for the time being. For she is committed to taking whatever steps will help her progress toward her future $\varphi$-ing. In other words, at $t_{1}$ she intends protempore to either $s_{1}$ or $s_{2}$ or . . or $s_{m}$ at $t_{2}$ (which are ultimately coimpossible implementations). As she gathers additional relevant information, she might decide before reaching the c-point at $t_{2}$ which of these disjuncts to pursue. But her decision might wait as late as $t_{2}$. Either way, this shows that any partial plan has the same structure as a PTD intention.

4.3 The only notable difference is that in the case of the partiality 
of implementation, the PTD sub-plan is subsumed under a larger and longer plan, which need not be disjunctive. In this scenario, the PTD sub-plan about what to do at $t_{2}$ is subsumed under the plan to $\varphi$ at $t_{3}$. As a result, the effects of the choice among the disjuncts is not expected to amplify downstream, but rather to be "reabsorbed" soon, since it is a matter of local implementation of one's continuous advance toward the future $\varphi$-ing.

4.4 The disjunctive character of PTD intentions becomes more dramatic when the choice is supposed to amplify after the c-point. But this amplification is not a necessary feature of PTD intentions. Likewise, in many cases of partiality, the costs of keeping open the mutually incompatible disjunctive sub-plans might be much less severe than in the standard PTD intentions, since the sub-plans are ultimately alternative ways of implementing the same goal, which is set by the master plan. Even so, the differences between paradigmatic cases of partial plans and paradigmatic instances of PTD intentions are not due to a different underlying structure. Both cases share the basic structure of pro-tempore disjunction, even if the structure can be differently instantiated depending on the specific forces that one's goals and circumstances exert on both the upstream and downstream stages of one's pursuit.

\section{Partial Intentions}

5.1 Richard Holton (2009, Ch. 2) has recently suggested that a distinctive kind of attitude - a partial intention - is required to handle scenarios similar to the one discussed here. In Holton's example, I intend to remove a tree brought down by a storm. There are several possible ways of achieving this end $E$ : I can lever the tree with a crowbar, saw it with a chainsaw, drag it with a rope, or call the local tree company to move it. These options are eventually incompatible. But I do not yet know which is better. For the time being, I want to keep all of them open, which might force me to bear the costs of multiple preparatory steps.

This scenario falls between the paradigmatic cases of partial plans and those of PTD ones. The options are ways of implementing a single master end $E$ (like in a partial plan), but the 
costs of keeping them open are higher than in simple partiality, whence the resemblance with standard PTD intentions. As I have argued above, the difference between these two scenarios is a matter of degree. Hence, I will consider Holton's proposal as it applies more generally.

5.2 Partial intentions are intention-like attitudes. As Holton (2009, 35) writes: partial intentions "play the same roles in curtailing deliberation, resolving indeterminacy, and enabling coordination that intentions play: you fix on a small number of plans from the many that occurred to you and that you might have pursued, and as a result of this you can coordinate around your other plans [...] and with other people." What is distinctive is that partial intentions, unlike all-out ones, are not subjected to the pressure for strong consistency. An all-out intention is supposed to be consistent both with the agent's beliefs and her other intentions. But a partial intention is only subjected to a weaker requirement: If one partially intends to $\varphi$, one is required to have a partial belief that one will succeed in $\varphi$-ing (or at least one is required not to all-out believe that one will fail at $\varphi$ ing)—-see Holton (2009, 41-6).

5.3 In support of his account, Holton often appeals to the parallel between partial beliefs and partial intentions. But given the controversial nature of partial beliefs and the limitations of arguments from analogy, I will set these considerations aside.

Additional support comes from an argument by elimination. Holton claims that all-out intentions with complex contents cannot account for these scenarios. First, we can't appeal to allout conditional intentions since no specific conditions are attached to alternative options. Second, all-out disjunctive intentions lack appropriate explanatory force. It is only when the all-out disjunctive intention is broken down into its components (the alleged partial intentions) that the agent's specific actions can be explained. As Holton $(2009,38)$ writes: "It is my partial intention to get the tree company to move the tree that causes me to phone them; if we are limited just to all-out disjunctive intentions, we can give no explanation of this."

5.4 I agree with Holton that explanatory force is crucial. The issue arises even when the incompatible options are not 
subsumed under a single end $E$. For a standard PTD intention, Holton's concern is that an action such as my making flight reservations to Austria could be explained by the partial intention to go to Austria, but not by the all-out PTD intention to go either to Austria or to Italy.

5.5 To see where the problem might lie, consider the explanatory force of a categorical all-out intention. Imagine that I categorically intend to get the tree company to move the tree. It is uncontroversial that, if I am rational, this intention might explain my phoning the tree company now. This is so when the categorical intention is non-deviantly combined with my current belief $B$ that (1) phoning the company now is a necessary means to get the company to move the tree, and that (2) I have now the ability and opportunity to phone the company.

Consider now a partial intention to get the company to move the tree. This intention, when non-deviantly combined with the belief $B$, appears as apt as the categorical intention at explaining my phoning now. Its partiality makes no difference to its explanatory power with respect to my phoning. The partiality rather affects which belief is rationally justified on account of my intention: I am not justified to all-out believe that I will get the company to move the tree, since I might end up handling the tree in another manner. But, Holton would claim, I am justified in holding the partial belief that the company is going to move the tree. So far, so good.

5.6 Consider now the PTD intention to either call the tree company or saw the tree. Does this intention lack explanatory power? If I phone the company, this action can be explained by combining the PTD intention with the same belief $B$ that I now have the ability and opportunity to take the necessary means. The disjunctive and pro-tempore character of the intention does not seem to make any difference to its explanatory force. Given the instrumental fit between phoning the company and getting it to move the tree, it seems that the PTD intention together with $B$ can explain one's action in the same way in which a categorical intention (either all-out or partial) does. ${ }^{7}$

5.7 Even so, one might suggest on Holton's behalf that in a PTD plan the explanation often goes through an intermediate step: in 
order to implement the plan, the agent might first focus on one of the disjuncts and only then considers what she is to do to make the disjunct advance. For instance, I might be moved to phone the company not directly by the PTD intention but by my focusing on the sub-plan of getting the company to come. It is only because I am now thinking about getting the company to come that the belief $B$ together with the intention explains my action of phoning the company. Or so one might argue.

If this is the correct explanation, one might then ask what kind of attitude as a rational subject I have toward the disjunct that is at the focus of my attention. It seems that I cannot say that I all-out intend to get the company since this intention would not meet the demand of global consistency. Holton's suggestion would come to the rescue: what I have is just a partial intention, which is not under the pressure of global consistency.

5.8 This is a radical solution. It asks us to acknowledge a novel kind of attitude. Before going this route, however, we must make sure that we cannot accomplish something comparable by relying on familiar all-out intentions, albeit ones with a complex content. If I have to explain why I am phoning the company, it seems that I can appeal to my intention to get the company as one of thealternative options that I am still pursuing right now. This is the option on which I am focusing my attention right now without having thereby acquired a novel kind of attitude. I have a standard all-out intention with a content that is qualified as one of the open sub-plans of a larger project.

This is a very familiar kind of sub-plan, one in which we often engage when implementing a PTD plan. But this sub-plan does not commit me to believing that I will succeed in it, since I appreciate how the continuous pursuit of the sub-plan ultimately depends on the fate of the other options.

The content of my present all-out intention directed at getting the company has a complex structure. But this complexity just reflects the contribution of this sub-plan to the PTD intention. I intend all-out to get the company as the alternative on which I am presently focusing among the various eventually co-impossible options (which I am still trying to keep all open for the time being) to remove the tree. This a perfectly 
fine content for an all-out intention. One that we are very familiar with even if not necessarily one that we would usually express in this form.

5.9 Hence, it is no objection to my analysis in terms of a complex content of an all-out intention (rather than of simple content of a partial intention) that the intentions toward a subplan of a PTD plan are often expressed categorically. How much complexity one is going to express is ultimately a matter of the pragmatics of communication. If all that $\mathrm{I}$ am trying to communicate to you is how my phoning makes any instrumental sense, it might be sufficient to say: "I intend to get the tree company." But if you are the one who is supposed to provide me with the saw that I need for the alternative sub-plan, I will be more inclined to spell out the complexity of my plan. I want to avoid any troubling misunderstanding, including your giving the saw to someone else. So I might say to you something like: "I am phoning the tree company because I want to keep open the option of their coming to get the tree but I have not yet made up my mind about which is the best way to remove the tree... so please still lend me the saw."

5.10 I have two additional serious reservations about Holton's proposal. First, there should be a methodological presumption against the proliferation of novel kinds of attitudes, especially on the face of the availability of alternative accounts in terms of complex contents. If my previous considerations are correct, there is no reason to favor introducing a distinctive kind of attitude - a partial one - directed at an unqualified content rather than sticking with standard, all-out, attitudes directed at a complex content.

5.11 Second, turning Holton's objection on its head, I want to argue that partial intentions are explanatorily weak. Although a partial intention might explain some of the steps of implementation, it cannot explain how these steps figure into any larger plan and how this might affect their nature and timing. Phoning the tree company might be explained by the partial intention to get the company, but the fact that I am phoning now rather than later, say, might have to do with how this action fits with my other concurrent sub-plans. As far as getting the company to come, I might have called at a different time. But I 
have to call now given that at the other times I will be busy preparing for the alternative options I am still keeping alive. The partial intention cannot explain the timing of my call, since these other sub-plans do not figure into the content of the partial intention.

True, the partiality of the intention hints at the existence of other eventually incompatible sub-plans. But it does so only in a generic form that does not help explain the specific unfolding of the agent's conduct in response to the actual character of these sub-plans. Likewise, a partial intention offers no explanation of why one might find advisable to give up that sub-plan in favor of another one. A partial intention, at most, warrants only a partial belief in its future success but it is silent on what might stand in the way of its being carried out, that is, it is silent on the specific character of the alternative sub-plans. Finally, a partial intention is not under any rational pressure to handle the competing sub-plans. Hence, except for those times when the agent does not face any immediate interference from the sub-plans, a psychology that operates through partial intentions rather than all-out intentions is in danger of being ineffective.

5.12 The partiality only shows in the irrationality of an all-out expectation about future success but it does not affect the rationality of the agent's conduct in the pursuit of the partially intended goal. This deprives the partial attitude of its explanatory force. If the psychological work were really done by the partial attitude, we should expect the rational agent to be oblivious to the existence of the competing projects while carrying out any specific partially intended goal. But this is exactly what we do not expect of such an agent. The only way to give a full explanation is to revert to an all-out intention directed at a complex disjunctive content. It is only at that level that the pressure for global practical consistency is exerted, a pressure that plays an indispensable role in any perspicuous psychological explanation of the agent's conduct.

5.13 To sum up, I see no benefits in preferring an account in terms of a partial attitude toward a simple content, rather than an all-out attitude toward a complex content. The resources to explain the conduct of the rational agent are to be found in the complexity of the content. Much work still needs to be done to spell out the 
implications of the complex contents of intentions both for practical rationality and for psychological explanation. But I have not yet found compelling reasons to despair that this job can be successfully carried out without introducing any partial practical attitude. ${ }^{8}$

\section{References}

Bratman, M. E. (1987). Intentions, plans, and practical reason. Harvard University Press.

Bratman, M. E. (2008). Intention, belief, practical, theoretical. In Timmerman, J., Skorupski, J., \& Robertson, S. (Eds.), Spheres of reason (pages 29-61). Oxford University Press.

Bratman, M. E. (2012). Time, rationality, and self-governance. Philosophical Issues, 22.

Ferrero, L. (2009). Conditional intentions. Noûs, 43(4), 700-741.

Ferrero, L. (2010). Decisions, diachronic autonomy, and the division of deliberative labor. Philosophers' Imprint, 10(2), 1-23.

Ferrero, L. (2014). Diachronic structural rationality. Inquiry, 57(3), 311-336.

Ferrero, L. (2015). Ludwig on conditional intentions. Methode.

Holton, R. (2009). Willing, wanting, waiting. Oxford University Press.

Klass, G. (2009). A conditional intent to perform. Legal Theory, 15(2), 107-147.

Ludwig, K. (2015). What are conditional intentions? Methode.

Yaffe, G. (2004). Conditional intent and mens rea. Legal Theory, $10,273-310$.

Yaffe, G. (2009). Trying, acting and attempted crimes. Law and Philosophy, 28(2), 109-162.

\footnotetext{
${ }^{1}$ For my view on conditional intentions see Ferrero (2009) and Ferrero (2015). See also Yaffe (2004), Klass (2009), and Ludwig (2015).

${ }^{2}$ For a discussion of intentions and underdetermination, see Ferrero (2010), Bratman (2012), and Ferrero (2014).
} 
${ }^{3}$ Normally, in undertaking co-impossible projects in the pro-tempore mode, an agent expects that if she is unable to determine which is better by the c-point, she will be indifferent between them at that point. When that happens, she can resolve the indifference by relying on any of the mechanisms that operate for standard disjunctive intentions of indifference. In some special cases, the agent might qualify her PTD intention by specifying what she would do at the c-point if she has not been able to determine which disjunct is best by then. For instance, she might even intend to pursue neither at that point.

${ }^{4}$ For further discussion of agglomerativity see Bratman (2008) and Yaffe (2009).

${ }^{5}$ For a discussion of how conditional intentions become moot, see Ferrero (2009, 7057) and Ludwig(2015).

${ }^{6}$ For a discussion of the transformation of a conditional intention into what I call a "circumstantially unconditional" one, see Ferrero $(2009,710)$.

${ }^{7}$ If the intention is disjunctive because of my indifference between getting the company to move the tree and sawing the tree myself, phoning might not be necessary to the intention's success. It is only when it is no longer possible for me to saw the tree that phoning the company becomes necessary for the success of the disjunctive intention. However, this does not entail that one is unable to explain the taking of nonnecessary means to the success of a disjunctive intention. One could explain this in the same way as one explains the taking of non-necessary means for either categorical all-out intentions or for partial intentions. Whatever additional elements, if any, might be required to complete the explanation for these intentions, these elements appear to be available for disjunctive intentions as well, both all-out and PTD ones. In any event, unlike the case of indifference, if I have a PTD intention, phoning the company might be a necessary means to the success of this intention even if sawing the tree myself is still an available option. For phoning is a necessary means to getting the company to move the tree, which is something that I am presently committed to, given that I am committed to keeping both options open for the time being.

${ }^{8}$ An earlier (and much longer) version of this paper was presented at talks at USC, Queens University at Kingston, and the University of Toronto. Thanks to the audiences, and especially to Philip Clark, David Hunter, Rahul Kumar, Lewis Powell, Sergio Tenenbaum, Gary Watson, George Wilson, and Gideon Yaffe. Thanks for stimulating conversations to Ray Buchanan and Luis Cheng-Guajardo. Thanks to Roman Altshuler and Michael Sigrist for their comments and editorial work. A special thank you to Gideon Yaffe who first prompted me to think about disjunctive intentions. 